\title{
Agôn
}

9| 2021

Rater

\section{À propos du pfüsch}

\section{Clémentine Cluzeaud}

\section{(2) OpenEdition}

Journals

Édition électronique

URL : https://journals.openedition.org/agon/8644

DOl : $10.4000 /$ agon. 8644

ISSN : 1961-8581

\section{Éditeur}

Association Agôn

Référence électronique

Clémentine Cluzeaud, «À propos du pfüsch », Agôn [En ligne], 9| 2021, mis en ligne le 16 juillet 2021, consulté le 20 janvier 2022. URL : http://journals.openedition.org/agon/8644 ; DOI : https://doi.org/ 10.4000/agon.8644

Ce document a été généré automatiquement le 20 janvier 2022.

Association Agôn et les auteurs des articles 


\title{
À propos du pfüsch
}

\author{
Clémentine Cluzeaud
}

1 Dans le langage des techniciens de l'Opéra du Rhin, à Strasbourg, le pfüsch est un terme alsacien qui désigne un travail bâclé1. "Ça, c'est (du) pfüsch », dit-on d'un tulle pas très bien tendu, d'un morceau d'adhésif qui sert à maintenir un objet mais dépasse d'un pendrillon, de la réparation précaire de l'accroche d'un tableau. Dans le pfüsch ${ }^{2}$ se manifeste une entreprise tout à fait particulière. Il est le travail réalisé mais raté : il peut tenir le temps d'une séance quand on est dans le jus. Au-delà de ce terme somme toute très régionaliste - sans doute trouverait-il ses équivalents dans chaque maison d'opéra -, les métiers techniques du plateau à l'opéra se manifestent comme l'interdiction faite à la technique de rater, et davantage encore, de faire voir le ratage au public. Elle ne le supprime évidemment pas. Mais les ratés techniques sont d'autant moins admis qu'ils sont, pour un certain nombre d'entre eux, prévisibles, prévus, et de ce fait préparés. Un certain nombre de règles balise ainsi l'entreprise du spectacle : le service de sécurité vérifie l'inflammabilité des matériaux, les chargés d'étude testent la résistance des décors, on règle la prise en main d'un objet, on déploie des consignes écrites autour d'une manutention particulière.

2 Plutôt que de faire le tour de ces consignes de sécurité qui tentent d'éviter les ratés mais se manifestent surtout comme une protection en direction des biens et des personnes (publics, techniciens) -, nous aimerions examiner les espaces et les moments où, à l'image du fameux pfüsch, le raté se lie à l'endroit d'une rencontre entre technique et artistique. Le pfüsch dessine en effet une série de frictions dans le monde très segmenté de l'opéra. Avant de parcourir la temporalité de la création d'un spectacle d'opéra en précisant certaines de ses apparitions, et notamment chez les accessoiristes, puisque c'est dans ce cadre que nous l'avons entendu pour la première fois ${ }^{3}$, nous préciserons quelques-unes des modalités du pfüsch, permettant de mieux cerner cette singulière pratique du raté. 


\section{De la répétition à la représentation : lexique, naissance et usages du pfüsch}

\section{Le pfüsch, un bricolage entre}

3 Rejoignant l'acception alsacienne, la langue allemande fait du Pfusch (sans tréma et avec une majuscule) le synonyme d'un travail maladroit, loupé ou délibérément mal exécuté. On ne sait s'il est une maladresse, une négligence, une faute délibérée, une tricherie ou une dissimulation, bref s'il revêt un caractère intentionnel ou non ${ }^{4}$. Le Pfusch rend visible l'opération technique en ne s'embarrassant pas de l'esthétique de sa réponse $\mathrm{e}^{5}$. Son origine serait onomatopéique: il traduirait le bruit produit par une poudre qui s'enflamme ou celui d'un tissu de mauvaise qualité qui se déchire ${ }^{6}$. Suivant cette étymologie, il est la réponse matérielle immédiate à un frottement, la manifestation d'un contact rappelant son intervention proprement contextuelle.

4 Le pfüsch trouve son équivalent dans d'autres secteurs. Il est le kludge dans l'argot des informaticiens. Créé pour arranger rapidement une difficulté, celui-ci fait parfois apparaître le "syndrome du plat de spaghetti », qui désigne péjorativement un code peu clair aux exceptions nombreuses. Il est une "verrue logicielle », " un assemblage temporaire (matériel ou logiciel) d'éléments hétéroclites, grossier et rudimentaire, conçu sans suivre les règles prescrites ${ }^{7}$ ». En biologie, ces termes rejoignent également l'approche méthodologique dite du quick-and-dirty ${ }^{8}$ qui recouvre un protocole vérifiant rapidement une hypothèse mais se passe des injonctions d'une expérience soigneusement préparée ${ }^{9}$. Le pfüsch, kludge ou quick-and-dirty sont autant perçus comme les produits d'amateurs astucieux qu'ils sont vécus comme "désolant $[\mathrm{s}]^{10}$ ", lorsqu'ils sont issus du travail d'un professionnel. Cette distinction entre une chose «bien faite » et une "malfaçon" se retrouve dans la Préface de Pratique pour fabriquer scène et machines de théâtre de Nicola Sabbattini ${ }^{11}$, dans laquelle Louis Jouvet relate ses débuts en tant que technicien de théâtre. Alphonse, le chef machiniste, sert de guide au jeune ambitieux. "S'il s'agissait d'une épissure sur un cordage, ou du dernier nœud à une équipe, à la fin de sa démonstration, Alphonse disait: "Voilà, t'as rien à te reprocher"12" ". Cette phrase, que Louis Jouvet précise retrouver de nombreuses fois dans l'ouvrage de Nicola Sabbattini, sous la forme "Ainsi aura-t-on fait ce qu'il fallait ${ }^{13}$ ", fait se manifester, en figure inversée et redoutée, celle du pfüsch, objet de critique possible ${ }^{14}$.

\section{Naissance d'un pfüsch}

5 À l'opéra, le pfüsch est vécu par les techniciens comme la conséquence d'un manque de temps. Il est une réponse technique qui se souhaite immédiate - et dont les aspects ne sont donc pas forcément maîtrisés - à une demande pressée de l'équipe artistique dans le temps de la répétition ou dans celui de la représentation. Pourtant, il est moins un manque de temps que le symptôme d'une superposition des temps et des espaces. Alors qu'à l'atelier se préparent les accessoires, les costumes et les décors, loin du bouleversement et de l'empressement du plateau ${ }^{15}$, le $p$ füsch, lui, émerge de la collision du temps et de l'espace commun entre le monde artistique et celui de la technique. Le pfüsch est un tiraillement : il naît de l'action du plateau, doit donc se résoudre au même endroit tout en évitant de prendre (faire perdre) du temps. De fait, il fait distinguer 
l'accessoiriste d'atelier de l'accessoiriste de plateau. Pour cela, une phrase de Georges Braque semble bien utile: "Ce qui est entre la pomme et l'assiette se peint aussi ${ }^{16}$ ». Nous pourrions concevoir l'accessoiriste d'atelier comme celui qui se préoccupe de la fabrication de la pomme et de l'assiette (dans les conditions projetées de l'action) alors que l'accessoiriste de plateau s'occupe de ce qu'il y a entre les objets, de leur action (les conditions réelles de l'action). Il est celui qui répondra par un point de colle ou un morceau d'adhésif double-face au problème d'une pomme qui roule sur la table, ou encore suggérera une solution au souhait d'un metteur ou d'une metteuse en scène de voir le fruit glisser sur le sol puis voler dans les airs ${ }^{17}$. L'accessoiriste de plateau est celui qui se préoccupe de l'entre-deux à imaginer, de ce qui ne peut se manifester qu'en jeu. Il est l'agent du situationnel, du « vide » créatif à investir. Il lui faudra bricoler une solution entre les usages, permettant ainsi de poursuivre la répétition ou la représentation: l'entre constitue à la fois l'instant et l'espace créatif de son travail. Ce technicien fait alors écho au bricoleur dont Claude Lévi-Strauss brosse le portrait dans son ouvrage La Pensée Sauvage :

La règle de son jeu [celle du bricoleur] est de toujours s'arranger avec les « moyens du bord ", c'est-à-dire un ensemble à chaque instant fini d'outils et de matériaux, hétéroclites au surplus, parce que la composition de l'ensemble n'est pas en rapport avec le projet du moment, ni d'ailleurs avec aucun projet particulier, mais c'est le résultat contingent de toutes les occasions qui se sont présentées de renouveler ou d'enrichir le stock, ou de l'entretenir avec les résidus de constructions et de destructions antérieures ${ }^{18}$.

6 Alors que l'ingénieur voit sa réussite dans l'adéquation entre le plan et la réalisation, Jérémy Liron, peintre et écrivain, suggère que le bricoleur est celui qui s'adapte, délaisse « la forme envisagée au profit de ce qui se décide comme ajustement bancal, intuitif et tâtonnant, incapable de certitude ${ }^{19}$ ", celui qui tâtonne, ruse. " Il abandonne sa capacité démiurgique au profit de [...] combinaisons fortuites entre éléments préexistants [...], d'aléas de l'exécution ${ }^{20} »$. Paradoxalement, le bricolage décrit donc l'abandon du contrôle en même temps que la découverte de la technique. Il est la «science du concret» formalisée par Claude Lévi-Strauss et fait écho à l'habileté ( «skill») définie par Tim Ingold comme " comme créativité dans l'acte de production lui-même ${ }^{21}$ ». Celle-ci est une attention prêtée à "la création d'opportunités de perception et d'action ${ }^{22}$ ", engageant le corps dans son entier et l'environnement avec lui. Elle réfute la logique hylémorphique, «cosa mentale » antérieure à la réalisation ${ }^{23}$. Le pfüsch est ce bricolé qui ne suit pas les règles du savoir et désigne davantage une solution trouvée en action. Il n'est pas du côté de la scénographie pensée, mais bien du geste qui résout une situation imprévue. C'est encore Louis Jouvet, racontant son maitre Alphonse, qui montre combien la machinerie échappe, elle aussi, aux lois mathématiques.

[U]n après-midi où ma science de la géométrie l'avait particulièrement exaspéré, il [Alphonse] m'a mis au défi de tracer une coupe à 45 degrés sur le tronc cylindrique d'un tuyau de poêle. Ce fut une honte et un émerveillement pour moi quand Alphonse, plus laconique que jamais, après avoir longtemps considéré mes essais prétentieux et ridicules, apporta un seau d'eau et un fil à plomb et fit tremper, incliné à l'angle voulu, le tuyau dans le plan horizontal du liquide.

«Le théâtre, m'a-t-il-dit, c'est pas de la géométrie: jouer un rôle, c'est pas de la mémoire et la mécanique au théâtre, c'est pas de la mécanique ${ }^{24}$. » 


\section{Outils du pfüsch}

7 Le pfüsch, à l'instar du bricolage, devient l'art de l'agencement et de la composition en action dont les méthodes «sont avant tout combinatoires ${ }^{25}$ ». Un petit nombre de techniques simples et un répertoire limité d'outils suffisent. C'est dans l'espace des découvertes que se lit plus précisément ce répertoire. «Dans le vocabulaire théâtral, le terme de "découverte" indique l'écart dans le décor qui laisse apercevoir la coulisse. Mais, il est également employé pour désigner le châssis placé en cet endroit pour masquer la faille ${ }^{26}$. Chantal Guinebault-Szlamowicz souligne avec cette définition le paradoxe des découvertes. Alors que le premier sens désigne la manifestation de «la théâtralité, la mise en œuvre du spectacle, l'envers de la représentation ${ }^{27}$ ", le second se charge de l'occulter et de rétablir l'illusion. C'est sur ce seuil, cet entre, que s'établissent les limites entre le montré (au public) et le caché (des coulisses), entre le spectacle et la technique : dès les coulisses passées, le danseur s'écroule, le chanteur crache dans son mouchoir. Le pfüsch en tant que réponse technique serait conventionnellement du côté des coulisses, mais sa précarité et sa possible visibilité jouent sur l'équilibre fragile des découvertes. Autour de celles-ci s'organise alors une véritable activité de rafistolage que l'on pourrait qualifier, elle aussi, de pfüsch. L'ensemble de l'équipe technique coiffeurs et maquilleurs, habilleurs, accessoiristes, machinistes - attend l'interprète pour reprendre un accessoire, remettre en place une mèche de cheveux, recoudre en vitesse un bouton qui a lâché. Le pfüsch est une réponse rapide : il doit s'accorder à la temporalité de la sortie de scène de l'interprète et tenir le temps de son apparition sur la scène. Ce moment d'urgence signe la distinction entre la haute technicité déployée par les ateliers de fabrication et une certaine forme de débrouillardise, parfois moquée, des métiers du plateau. Se matérialisent à cet endroit les indispensables instruments aux réparations urgentes, « répertoire d'outils » du bricoleur. Les coiffeurs-maquilleurs portent, attachés à leur ceinture, un paquet de mouchoirs, de la laque, un peigne et des barrettes ; les habilleurs, leur porte-épingles au poignet et les aiguilles déjà enfilées à des fils de différentes couleurs pour réajuster un bouton ou coudre un ourlet. Les accessoiristes, quant à eux, se tiennent prêts avec toutes sortes d'adhésifs attachés à leur ceinture. L'adhésif large noir désigné par l'anglicisme gaffer ${ }^{28}$ est un essentiel. Pensé pour se rendre à la fois résistant et invisible, ce ruban noir, mat, ne brille pas sur la scène, se coupe à la main - il n'a donc pas besoin d'autre outil -, il est capable d'une bonne adhérence tout en ne laissant pas de trace de résidu une fois enlevé. C'est donc l'outil parfait d'un bon pfüsch qui se voudrait invisible et incassable ${ }^{29}$. Il est complété par l'adhésif double-face, se réclamant d'une totale invisibilité et d'une rapidité redoutable, et donc accordé au pfüsch de la représentation. Le gaffer blanc constitue également un outil indispensable. Il signale la frontière entre ce qui est visible du public et ce qui reste à l'abri de son regard et prévient l'équipe technique des potentiels dangers de coulisses plongées dans l'obscurité.

8 Là où l'opéra et le théâtre préfèreraient le vite fait bien fait surgit parfois ce frère repoussant, ce pfüsch mal aimé. Déviation du modèle exemplaire, piraterie irréconciliable avec un savoir-faire transmissible, il est pourtant le bricolé nécessaire aux temps et aux espaces communs de l'équipe artistique et technique. Mais il peut également se manifester de façon tout à fait particulière dans le temps de la répétition : il rend alors compte de l'ambiguïté de la nature et de la valeur de l'objet durant la création. 


\section{Le Grand pfüsch de la répétition}

\section{Le plateau de répétition comme esquisse}

9 Les premières répétitions d'une production d'opéra se déroulent généralement dans une salle annexe au plateau ${ }^{30}$. Les machinistes tentent d'y recréer les volumes suggérés par les plans de la scénographie, en attendant la livraison des éléments de décors finis. Le plateau scénique est délimité par des lignes d'adhésif blanc, qui marquent autant la frontière entre ce qui sera visible sur le plateau et les futures coulisses du spectacle que la distinction entre l'espace scénique et l'espace contextuel. Pour toutes les créations en répétition, les mêmes éléments sont réutilisés. Des petits escaliers noirs qui mènent à des praticables forment des aires de jeux surélevées, quelques châssis dessinent des murs, des cadres de porte indiquent des entrées. Cette simulation ${ }^{31}$ constitue des appuis de jeux fondamentaux pour les chanteurs et les figurants. Il conserve, si la salle le permet et autant que faire se peut, les écarts de distance entre les objets pour tenter de construire des repères dans la partition. Le marquage à l'adhésif signe les récits de construction des espaces, les emplacements des objets et la modélisation de la dramaturgie : à chaque couleur d'adhésif correspond un acte. Le plateau de répétition ressemble à une maquette à échelle $1: 1$ rudimentaire.

\section{L'objet pfüsch : précaire ou labile?}

10 Ainsi le plateau de répétition devient-il le royaume du pfüsch. Le raté recouvre ici l'idée d'un raccourci, suffisant dans l'instant de la répétition mais insuffisant pour la représentation : il est le précaire et le sans-finition qui s'accorde à l'attente du véritable décor. Durant les répétitions de l'opéra Beatrix Cenci mis en scène par Mariano Pensotti, les sculptures de chiens trônant dans la demeure des $\mathrm{Cenci}^{32}$, en construction dans les ateliers, sont ainsi allégrement remplacées par des cartons de déménagement sur lesquels est écrit le mot «chien» au marqueur noir sur un morceau d'adhésif blanc ${ }^{33}$. L'arc d'un indien trouvé dans un magasin de jouet et tenu par un chanteur en jeans suppose Cupidon et son arc dans La Divisione del Mondo ${ }^{34}$, un gobelet en plastique simule la tasse de thé d'un comte que l'accessoiriste s'empresse d'aller chercher.

Louis Jouvet rapporte la même expérience lors de ses répétitions :

[...] un paravent délabré [...] figurera un mur, quatre marches boîteuses [...] suggéreront le gigantesque escalier, dont les acteurs n'auront que plus tard le bénéfice et la gêne ; leur offrir l'objet supposé qu'ils utiliseront à la représentation, vieux gobelet bossué qui sera une coupe d'or ciselé, vieux bâton qui représentera le glaive ${ }^{35}[\ldots]$.

Durant ce temps et dans cet espace, le pfüsch, bien qu'il puisse apparaitre comme un raté aux yeux d'un visiteur non averti, s'en distingue : il est l'acceptation par tous d'un remplacement temporaire. Il devient l'effet d'une persuasion commune. C'est donc paradoxalement au moment le plus intense de la création que l'on ferme les yeux sur tout $^{36}$. Le «finement produit » est remplacé pendant six semaines de création par du pauvre et du précaire. Il est le remplaçable mais aussi le façonnable. Soumis à tous les tests - surtout ceux dont on se préviendrait avec le « véritable» objet - il prévient la rupture, construit les possibles du futur objet, ajoute des éléments trouvés dans le jeu. L'espace de répétition relève non pas tant du manque mais de la créativité que celui-ci provoque, ce qui n'est pas sans rappeler les usages de l'objet pauvre décrits par Jean- 
Luc Mattéoli ${ }^{37}$. L'auteur distingue trois catégories d'objets : la première regroupe les objets invisibles et dont l'existence, issue du jeu du comédien, ne concerne pas l'accessoiriste. La seconde recouvre les objets indiciels. Une table de café évoquerait ainsi une brasserie dans son ensemble et contribuerait «à faire exister d'autres objets, absents mais liés comme lui au même paradigme ${ }^{38}$ ». Dans la salle de répétition, ces objets indiciels sont remplacés par une matérialisation précaire : un châssis, une table et deux chaises dépareillées évoquent une brasserie et sa porte d'entrée. Ainsi, le pfüsch pourrait désigner l'esquisse d'un objet qui se veut déjà incomplet. La troisième catégorie d'objet pauvre est fondée sur la métaphore.

Un objet quelconque, manipulé par un acteur, change de nature pour un instant par la grâce du jeu : le chapeau d'Arlequin, rentré dans le col du vêtement, devient la serviette d'un repas improvisé, et sa batte, glissée entre les jambes, un phallus surdimensionné quand passe une jolie servante ${ }^{39}[\ldots]$.

Comme l'objet métaphorique de Jean-Luc Mattéoli, l'objet pfüsch est la manifestation d'un effort d'imagination à produire pour le spectateur. Mais alors que l'objet décrit par le chercheur devient visible par un «ensemble de gestes et de mimiques » de l'acteur, l'objet pfüsch, est, là encore, la métaphore d'un autre absent de la scène. Avant d'être ce chapeau d'Arlequin qui deviendra serviette, il sera peut-être n'importe quel chapeau de feutre "sous la main» choisi par l'accessoiriste. Indice d'un indice, métaphore d'une métaphore, l'objet pfüsch dessine un écart supplémentaire à l'objet de représentation. Il est un brouillon qui, paradoxalement, éloigne de l'idée finale du metteur en scène tout en lui permettant de mieux en éprouver les possibles. L'objet pfüsch devient l'esquisse indispensable au dessin final de l'objet. Les propos d'élie Konigson dans l'article "Espaces dramatisés " nous permettent de compléter cette réflexion. Le chercheur désigne trois objets de représentations qui caractérisent le modèle théâtral au cours des siècles : le plateau, l'ouverture et la sedes (la chaise) ${ }^{40}$. Il expose ainsi la transformation de l'objet matériel en objet de représentation sur l'espace du plateau :

D'objet matériel, qu'elle [la chaise sur la scène] continue d'être, d'ailleurs, elle se transforme en écho d'une réalité décalée, différente. D'objet quotidien elle se mue en objet de représentation - ville, pays, trône -, etc. En ce sens aussi, l'objet de représentation se différencie de l'accessoire qui, même faux, n'outrepasse jamais le sens manufacturier qu'il remplit hors théâtre. Un faux poignard, celui d'Oreste dans Andromaque, ne signifiera jamais autre chose qu'un poignard entre les mains d'un héros jaloux ${ }^{41}$.

L'objet manufacturé transcende sa matérialité et se mue en réceptacle d'imaginaire, à l'instar de l'objet pfüsch. Cependant, ce dernier - premier objet de répétition - ne distingue pas aussi nettement que le fait Élie Konigson l'accessoire de l'objet réel déposé sur la scène : il rend davantage compte d'opération de transfigurations entre les deux. L'objet ne connaît pas seulement une mutation, mais bien plusieurs. Le pfüsch manifeste la présence d'objets premiers, brouillons notoires, qui engagent les suivants, et qui peuvent être tout autant tirés du réel que déjà accessoires issus d'une précédente fiction. À cette occasion, le pfüsch révèle le contexte de production et les détours de la construction d'un spectacle au sein même de l'espace réservé à la représentation, rendant la frontière mimétique poreuse aussitôt le processus de création engagé. Lors des répétitions, il semble important de repréciser régulièrement, entre les équipes techniques et artistiques, la valeur de l'objet pfüsch. Car celui-ci, remplaçant sur le banc de touche dans l'attente de l'arrivée de l'objet titulaire, n'est pas sans pouvoir. L'objet 
substitut peut se révéler, au fur et à mesure des répétitions, être celui qui convient, en tout ou partie.

Durant le temps de la création, le pfüsch peut donc être toléré. Sa précarité devient une labilité. Il est l'esquisse qui se construit au fur et à mesure et rend compte de la mobilité de la recherche. Ces caractérisations du pfüsch engagent un trouble nouveau: l'idée d'une distinction entre la création de la scénographie d'opéra, souvent perçue comme une réalisation a priori des répétitions, et la construction de l'espace dans le temps de la création, suggérée parfois par les écritures de plateau, n'est plus aussi évidente. Il n'y aurait finalement que des situations intermédiaires, qui doivent composer entre ces deux approches.

Mais l'objet pfüsch ne peut durer : en ne répondant pas aux règles du savoir-faire, sa fragilité rend d'autant plus forte les risques d'accidents intrinsèques au spectacle vivant. Sa fabrication grossière et inesthétique le rend possiblement repérable depuis la salle, lors des représentations. Il manifeste alors la fabrication de l'illusion - et l'annule par le même mouvement. L'accessoiriste (et ses chefs) ne désirera qu'une seule chose : le remplacer. Ainsi, le pfüsch en tant que rater issu du processus de recherche semble admissible mais il ne peut s'établir sur la scène au risque du raté (ou ratage). Rattraper des ratés au risque d'en devenir un... Voilà la beauté de la logique pfüsch. Pourtant, dans le temps spécifique de la représentation, certains gestes techniques en font réapparaître les figures sur la scène.

\section{Pfüsch en représentation}

\section{Rendre visible}

La tricherie - on en revient à la définition du pfüsch - est au cœur du métier d'accessoiriste. La plupart des objets ne sont qu'une semblance des objets tirés du réel: la marmite sur la scène n'est pas en fonte, les livres de la bibliothèque sont en carton, le verre brisé en résine, la sardine à manipuler en silicone, etc. Ces trompe-l'œil recèlent des avantages: ils autorisent une manipulation plus aisée, permettent leur réutilisation, allègent leur poids et garantissent une certaine sécurité. Mais ce truquage a une limite : il n'est pas question qu'il se révèle au public. L'espace théâtral nourrit ainsi une forte distinction entre le visible offert au public et celui du travail technique : de façon conventionnelle, le visible sur la scène doit requérir l'invisible de la technique. Pourtant, parfois, sur la scène, s'articule un jeu autour de la manifestation de la technique. Dans ce cadre, le pfüsch pourrait s'entendre comme une sorte de refus du «tout esthétique » et de l'illusion totale. Il serait alors ce qui compose une trace de la technique à l'intérieur de la représentation. À ce sujet, un souvenir particulièrement éloquent : parmi les accessoires d'une production d'opéra issue d'une autre maison et que nous devions reprendre, se trouvait la lettre calligraphiée attribuée à un personnage. Mais sous l'écriture appliquée de la fiction se dessinait l'angoissante attente de l'accessoiriste. La missive, adressée à cette "Chère Pure Invention » était signée d'un « Non-sens absolu».

Non-Sens Absolu

10, Heures Quarante-cinq

Un mardi matin ${ }^{42}$

$[\ldots]$ 
Chère Invention pure,

C'est avec la plus grande apathie que je me trouve debout dans le bureau des accessoires du Théâtre de [...] en train d'écrire cette lettre des plus inintéressantes, qui ne dit rien, ne signifie rien ni aujourd'hui ni plus tard et n'aura aucune incidence sur quoi que ce soit.

Je resterai votre serviteur obéissant jusqu'à ce que mon contrat de travail prenne

fin - plus que quatre jours !

Cordialement,

Non-Sens Absolu ${ }^{43}$

Le pfüsch, non loin du travail en perruque, devient alors une prise de risque, un détour facétieux du technicien dévoilant l'envers du décor sans que le public ne puisse le voir. Caché sous l'illusion d'une écriture déguisée comme ancienne ${ }^{44}$, l'accessoiriste ne choisit pas un texte de remplissage à l'image du lorem ipsum utilisé par les graphistes ${ }^{45}$. Il offre bien plutôt un récit cocasse de sa position, exposé uniquement aux yeux de ceux qui partagent avec lui l'envers de la scène. L'apparition, au plateau, est régulée par l'ajustement de la distance au public, qui lui permet d'offrir un objet crédible à la fiction. Entre l'objet de représentation et son sens s'opère la même distinction qu'entre un signifié et un signifiant. L'incarné de l'écriture et l'incarnant de la lettre se lient à l'endroit de l'accessoire fabriqué. Cette lettre n'est pas sans rappeler les scholies des moines copistes du Moyen-Âge qui, dans les marges des manuscrits, ajoutaient parfois quelques commentaires bien plus pragmatiques que leurs écrits sacrés. Parmi ceux-ci, on retient, avec l'historien Michael Camille, une prière : « C'est fait, j'ai reproduit l'intégralité de la chose : pour l'amour du Christ, filez-moi à boire ", l'expression d'un soulagement : " Dieu merci, il fera bientôt noir », une plainte : «Oh, ma main » ou une simple constatation: "Ce parchemin est poilu ${ }^{46} »$. Trahissant la main de l'ouvrier, la marge, qu'elle soit celle de la page ou celle de la scène, manifeste les conditions de la réalisation de l'objet. Elle devient un jeu transgressif sur le montré et le caché, la fabrication de l'illusion et l'illusion du spectacle. Risque pris par l'accessoiriste, avec ou sans la complicité de l'interprète, l'objet se révèle être un espace de négociation tant dans le rapport à la fiction représentée que dans celui du travail.

\section{Braconner}

Avouons-le: le pfüsch est parfois en effet le lieu d'une faille dans les valeurs hiérarchiques de l'opéra, un pied-de-nez aux chefs de service ${ }^{47}$. Il est la ruse pour effectuer une tâche au plus vite. Il est le " pas vu pas pris ». S'il est découvert, il sera considéré comme une nuisance du fait de sa précarité et/ou de sa visibilité ; il faudra le défaire et le refaire autrement $:$ il est donc considéré comme une perte de temps ${ }^{48}$. Par ce tissage entre contexte et ruse ${ }^{49}$, le pfüsch rappelle fortement la tactique définie par Michel de Certeau, qui s'oppose à la stratégie. Cette dernière est désignée comme :

le calcul des rapports de forces qui devient possible à partir du moment où un sujet de vouloir et de pouvoir est isolable d'un «environnement ». Elle postule un lieu susceptible d'être circonscrit comme un propre et donc de servir de base à une gestion de ses relations avec une extériorité distincte ${ }^{50}$.

Suivant ce cadre, les réflexions, les prévisions, les précautions et le savoir-faire dont s'assurent les ateliers de construction sont autant de stratégies. Elles relèvent à la fois d'un rapport de domination mais aussi d'un usage théorique : le plan de bataille est une stratégie a priori quand le champ de bataille peut, lui, donner lieu à des tactiques. Le 
pfüsch surgirait à l'aune d'une tactique aventureuse, dans le temps de la création : sur le champ de bataille.

La tactique n'a pour lieu que celui de l'autre. Elle s'y insinue, fragmentairement, sans le saisir en son entier, sans pouvoir le tenir à distance. Elle ne dispose pas de base où capitaliser ses avantages, préparer ses expansions et assurer une indépendance par rapport aux circonstances ${ }^{51}$.

22 À l'inverse du «propre » stratégique, victoire du lieu sur le temps, le pfüsch « dépend du temps, vigilant[e] à y "saisir au vol" des "occasions" 52 ». Ce qu'il gagne, il ne le garde pas : le pfüsch ne peut devenir un savoir-faire. Il est un objet pratique $e^{53}$ à la lumière d'une situation qui le traverse. Il naît d' " une combinatoire d'opérations $s^{54}$ ", sans terrain propre. Mais il témoigne aussi d'un usage braconné : il atteste de l'usage réel - la tactique - face à l'usage prescrit, imposé par la hiérarchie et le savoir-faire - la stratégie. Il rend compte d'une appropriation. Il propose un écart d'avec les pratiques conventionnelles des corps de métier et offre des solutions nouvelles qui affirment un dynamisme de l'objet. En cela, il manifeste la rencontre imprévue des usages et peut être l'occasion d'une surprise, d'une invention. Plus encore, il met à mal l'idée que l'œuvre est pensée dans son intégralité, objet fini offert au public. Il invite à la percevoir comme un processus qui se construit aussi par des accidents et des hasards.

\section{Jouer}

Que se passe-t-il, dans le temps de la représentation, quand la figure du pfüsch se transforme en une action préméditée et volontairement visible ? Pour l'examiner, citons une autre de ces pratiques scéniques bien connue des usagers des coulisses: la blague de dernière, qui désigne une plaisanterie consistant à troubler son partenaire sur la scène et à tester sa capacité à ne pas rire sur le plateau lors de la dernière représentation. L'ajout d'une page érotique dans un livre que le comédien doit feuilleter, le remplacement de l'eau par de l'alcool ou l'ajout d'un accessoire impromptu sont des classiques du genre. La blague est jugée réussie si l'autre se retient de rire jusqu'aux coulisses. Elle se situe donc à cet ultime point de tension du caché et du montré: rien ne doit transparaître aux yeux du public. Comme la lettre de l'accessoiriste, elle est la manifestation d'une communauté qui se connait. Certains artistes contestent cette tradition qui fait de la dernière une représentation différente des autres. «J'estime que chaque spectateur, même celui qui vient voir la dernière, a le droit de voir le même spectacle. Donc pas de blagues de dernière ", a expliqué, un jour, un metteur en scène. Avec elle, l'illusion peut se rompre, la technique - qu'elle soit celle de l'interprète ou celle du technicien - se manifester, et avec elle vient se briser la crédibilité (la vraisemblance doublée de la réputation, pour ainsi dire) de la représentation.

\section{Étendre le pfüsch à l'œuvre}

Parfois, le geste technique devient l'événement d'une révélation publique. Alors que dans le théâtre postdramatique, ce phénomène peut apparaître fréquent, il reste encore très minoritaire à l'opéra. Lors des applaudissements finaux du Tour d'écrou de Benjamin Britten à l'Opéra du Rhin en $2016^{55}$, le metteur en scène Robert Carsen a souhaité que les machinistes retournent les châssis. Alors que pendant la représentation, ces châssis simulaient parfaitement les murs teintés de gris d'une 
maison bourgeoise, ils se révélaient soudainement comme un assemblage de bois brut sur lesquels le public pouvait lire des indications destinées aux techniciens. Pour de nombreux spectateurs, ce moment fut intense : il devint l'occasion de voir se révéler l'envers de la scène et de s'imaginer le processus de la représentation. Il rendait ainsi compte du thème de l'illusion émaillant le texte d'Henry James.

Certaines pièces élargissent cette intention à la pièce entière. En 2011, Philippe Quesne crée Pièce pour la technique du Schauspiel de Hanovre pour l'équipe technique permanente du théâtre. Il désire alors faire du travail des techniciens et de leurs interactions un véritable spectacle. Les machinistes apparaissent, soulèvent des décors, reproduisent les gestes quotidiens de la profession, discutent autour d'un objet. La mise en place de la scénographie par les techniciens fonde l'œuvre théâtrale elle-même. Cette création fait écho à une phrase de Jacques Copeau, dessinant son intérêt pour la réalité concrète du jeu des techniciens :

L'action réelle est belle sur notre scène. Le travail qu'y accomplissent les artisans, dans le mouvement qui leur est accoutumé, y paraît à sa place, cela vient de ce qu'ils font réellement quelque chose, [...] observent des temps réels [...]. L'acteur sur la scène, ne fait jamais rien réellement ${ }^{56}$.

La technique dessinerait donc la vérité de la scène. Le pfüsch, outre la manifestation de la technique comme événement, peut se révéler comme un parti pris esthétique en soi. L'artiste allemand Thomas Hirschhorn ou le catalan Jordi Colomer, avec leurs créations faites de cartons assemblés par de l'adhésif marron ${ }^{57}$, en seraient les figures de proue. Ce pfüsch pourrait alors bien correspondre au "réalisme opératif» décrit par Nicolas Bourriaud où l'œuvre ne cache plus sa technicité, et manifeste «la double appartenance au domaine du fonctionnel et à celui de l'esthétique ${ }^{58} »$. Le caractère pfüsch se retrouve aussi dans les hommages à l'aléatoire, à l'accident, aux objets trouvés et aux machines rouillées présents dans les spectacles de Camille Boitel, avec l'Immédiat (2009) par exemple, de Jean-Pierre Larroche ${ }^{59}$, ou de Pierre Meunier et Marguerite Bordat $^{60}$. Ces objets conservent les traces du contexte de création comme celles de leurs vies antérieures.

27 Figure singulière du raté, le pfüsch est la manifestation, intentionnelle ou non, des écarts qui composent la scène théâtrale. Il peut prendre plusieurs formes. Il peut désigner un essai bricolé, inesthétique durant le processus de recherche en répétitions. Il est une tentative qui peut rester un raté ou devenir une solution réussie. Il constitue également une réponse à l'urgence (et aux ratés) de la représentation, créant dans le meilleur des cas une astuce précaire, une illusion ténue, dans le pire, un raté malhabile. Enfin, le pfüsch pourrait s'entendre comme un raté volontaire, dans le cas d'une blague de dernière ou de la dénonciation d'une illusion. Il tisse son existence de la situation et manifeste la contextualité de la production du spectacle vivant. Sa précarité naturelle s'accorde au temps de l'éphémère du spectacle vivant. Il met aussi en danger cette production en appuyant son caractère fragile et en dénonçant, par sa visibilité, la fabrique de l'illusion. Mais le pfüsch est aussi une tactique. À cette occasion, il peut devenir un soulagement à la possible oppression de l'invisibilité du technicien. Le pfüsch organise alors des endroits d'intensité braconnés sur le territoire du plateau. Il est une capacité à inventer dans l'action et par l'action. Il peut être l'espace d'une liberté créatrice et joueuse. Équilibre précaire, son apparition peut faire événement mais aussi accident. Le pfüsch peut aussi ouvrir à la compréhension des enjeux matériels de la création autant qu'il peut être un point d'entrée d'une entreprise sociologique du spectacle. En effet, celui ou celle qui a déjà entraperçu les coulisses et 
les ateliers d'un spectacle comprend combien il serait intéressant de repenser son analyse à partir d'une culture technique et matérielle des œuvres et de l'analyse des gestes techniques qui l'accompagne. C'est d'ailleurs l'idée que développe Thomas Golsenne en proposant la notion de "bricologie », qui rendrait compte de l'œuvre contemporaine comme d'un «entrelacement de pratiques plus ou moins prévues ou accidentelles $^{61} »$. Il suit par là les pensées d'Henri Focillon, de Gilbert Simondon ou, plus récemment, de Tim Ingold, qui tentent de dénouer la mythification de l'œuvre d'art. Selon Thomas Golsenne, il est possible de s'aider pour cela du concept de « chaînes opératoires ", théorisé par André Leroi-Gourhan ${ }^{62}$, établissant étape par étape la fabrication matérielle de l'œuvre.

Grâce à l'emploi de l'outil « chaîne opératoire », on peut comprendre comment une suite d'actions, l'utilisation d'objets, qui, pris isolément, ne sont pas artistiques, permettent de produire pourtant une œuvre d'art. On appellera bricologie l'étude des chaînes opératoires qui mènent à la production d'une œuvre de création contemporaine $e^{63}$.

On ne peut que souhaiter que l'écart manifesté par le pfüsch se rende parfois davantage visible et qu'il puisse être un outil utile à une analyse «bricologique » du spectacle. Et qu'il atteste encore, bien sûr, de l'existence d'individus singuliers dans les coulisses.

\section{NOTES}

1. Dictionnaire alsacien-français Elsassisch en ligne, URL de référence : http://www.elsassisch.eu/ culture.alsace.pagesperso-orange.archive/page_p.html ; consultée le 5 octobre 2020. Edmond Jung, L'Alsadico, 22000 mots et expressions français-alsaciens, Strasbourg, La Nuée Bleue, 2006.

2. Prenant certaines libertés avec la langue alsacienne, nous utiliserons le terme pfüsch autant comme un adjectif que comme un substantif.

3. Depuis 2014, je travaille régulièrement comme accessoiriste plateau à l'Opéra du Rhin.

4. Sources: Harrap's universal Dictionnaire français/allemand, allemand /français, Conception et direction rédactionnelle Barbara Krüger, Nouvelle édition, Edimbourg, Paris, Stuttgart, 2008 p. 490. Grand Dictionnaire allemand Hachette et Langensheidt, équipe rédactionnelle du dictionnaire allemand-français Birgit Klausmann, Geneviève Lohr, Herbert Horn, Berlin, Munich, Langenscheidt, Paris, Hachette Education, 2009, p. 1208, entrées Pfusch, Pfuscharbeit, Pfuschen, Pfuscherei. Jacob Grimm, Wilhelm Grimm, Deutsches Wörterbuch, Leipzig 1854-1961, entrée Pfusch.

5. Nous renverrons ici le lecteur et la lectrice au pfusch am bau (que l'on peut traduire par " construction bâclée »), ensemble de solutions atypiques qui incite à douter du caractère conventionnel et réglementé prêté à certaines constructions en bâtiment. S'il s'avère que certains de ces pfusch am bau relèvent de la négligence de professionnels, on ne pourra qu'être épaté devant l'ingéniosité du cerveau humain, capable de résoudre des problèmes de manière aussi peu orthodoxes, voire complètement loufoques : le tuyau d'arrivée d'eau ne voit finalement pas d'inconvénient à traverser une fenêtre, une porte découpée suivant le siège des toilettes permettra la présence de WC dans un espace trop étroit. Rien n'est insoluble à qui le veut vraiment, nous dit le pfusch am bau, qui prête autant à rire qu'à se perdre dans l'infinie jungle des 
solutions du système D. On pourrait également reconnaître ses traces dans le livre Belgian Solutions de David Helbich, Anvers, Luster, 2015.

6. «Wohl zu futsch, ursprünglich lautmalend z. B. für das Geräusch von schnell abbrennendem Pulver oder für das Reißen von schlechtem Stoff », Dictionnaire Educalingo (Source des définitions : Oxford Languages), URL de référence : https://educalingo.com/fr/dic-de/pfuschen ; consultée le 5 octobre 2020. Jacob Grimm, Wilhelm Grimm, Deutsches Wörterbuch, Leipzig 1854-1961, entrée Pfusch.

7. Entrée «Kludge » ou "kluge », Office québécois de la langue française, URL de référence : http://gdt.oqlf.gouv.qc.ca/ficheOqlf.aspx?Id_Fiche=8873440; consultée le 5 octobre 2020.

8. Littéralement " rapide-et-sale », et généralement admis comme « rapide et efficace ».

9. À l'occasion de l'épidémie du Sars-CoV-2, cette approche a été largement débattue, entre les tenants du quick-and-dirty souhaitant répondre avant tout à une situation d'urgence, et les partisans d'une méthodologie plus lente mais dont les données répondent aux critères de la démarche expérimentale. Le quick-and-dirty a désormais sa résonnance dans le domaine du management dans lequel il est perçu comme la variable d'ajustement contextuelle à la situation de l'entreprise.

10. Entrée « Kludge » ou « kluge », Office québécois de la langue française, URL citée.

11. Louis Jouvet, Préface à Pratique pour fabriquer scène et machines de théâtre par Nicola Sabbattini [1638], Ides et Calendes, La Bibliothèque des Arts, Neuchâtel, 1994 [1942].

12. Ibid., p. XL.

13. Ibidem.

14. La nécessité du travail bien fait est de nouveau évoquée dans un passage de cette préface: «Un jour que je confiais à Camille [avec Léon, Camille est un des deux chefs machinistes de Louis Jouvet] l'impossibilité de monter une pièce et que je lui exposais les problèmes de machinerie insurmontables qu'elle présentait, il a répondu : "L'important, c'est que ce soit bien fait." ", op. cit., p. XXXV.

15. Nous tenons à présenter nos excuses à tous les constructeurs et constructrices en ateliers qui se sentent eux parfois pressés par les techniciens au plateau!

16. Henri Darasse, «L'Accident Pictural, à la lumière des concepts de François Jullien », dans François L'Yvonnet (dir.), Art et Concepts, Chantiers philosophique de François Jullien/ Ateliers d'artistes, Paris, P.U.F., 2020, p. 83.

17. Et même si, pour la répétition, l'envol de la pomme se fait au moyen d'une pomme de terre et d'un fil encore trop visible enroulé autour d'un balai, le tout dirigé par un accessoiriste que les découvertes ne cachent pas tout à fait...

18. Claude Lévi-Strauss, La pensée sauvage, Paris, Plon, 1962, p. 27.

19. Jérémy Liron, «Un soir de juin », Les pas perdus, Paris, France, 22 juin 2008 ; URL de référence : https://chapuisat.com/x/un-soir-de-juin ; consultée le 17 juin 2020.

20. Ibid.

21. Thomas Golsenne, "Les chaînes opératoires artistiques ", Techniques\&Culture, $n^{\circ} 64$, «Essais de bricologie. Ethnologie de l'art et du design contemporains », 2015, p. 29.

22. Ibid., p. 23.

23. La logique hylémorphique est entre autres décrite par Tim Ingold dans Faire, Anthropologie, Archéologie, Art, Architecture, Bellevaux, Dehors, 2017.

24. Louis Jouvet, Préface à Pratique pour fabriquer scènes et machines de théâtre par Nicola Sabbattini, op.cit., p. XL.

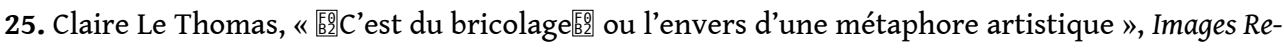
vues, Centre d'Histoire et Théorie des Arts, 2019, p. 9.

26. Chantal Guinebault-Szlamowicz, Scénographie, frontalités et découvertes dans le théâtre contemporain, thèse de doctorat, sous la direction de Luc Boucris, Arts du Spectacle, Montpellier 3, soutenue en 2002, citation extraite du résumé. 
27. Ibid., p. 16.

28. On peut noter qu'il se prononce gaffeur...

29. Parmi les conseils pour bien utiliser son gaffer, une règle élémentaire non dénouée d'humour : «Mieux vaut trop que pas assez ». Nous sommes effectivement loin d'un savoir-faire. URL de référence: https://faiteslepoint.wordpress.com/2012/02/25/lart-du-gaff-adhesifs-partie-1/ ; consultée le 5 octobre 2020.

30. Nous parlons depuis notre expérience d'accessoiriste à l'Opéra du Rhin. Sans doute d'autres maisons d'opéras offrent-elles des conditions de répétitions différentes.

31. Ce n'est pas tout à fait la Bauprobe, tradition principalement allemande, qui désigne le moment d'une simulation sur le plateau même de la future représentation.

32. Beatrix Cenci, d'Alberto Ginestera, opéra en deux actes, Livret de William Shand et Alberto Girri d'après les Chroniques italiennes de Stendhal et The Cenci de Percy Shelley, créé le 10 septembre 1971, au Washington Opera. La mise en scène présentée à l'Opéra du Rhin est de Mariano Pensotti, direction musicale Marko Letonja, décors et costumes Mariana Tirantte, création Opéra National de Rhin, saison 2018-2019.

33. Il sera donc possible de voir un chanteur enlacer ledit objet et caresser un angle du carton comme si c'était la truffe de l'animal.

34. La Divisione del Mondo, de Giovanni Legrenzi, Opéra en trois actes,

Livret de Giulio Casare Corradi, créé le 4 février 1675 à Venise. La mise en scène présentée à l'Opéra du Rhin est de Jetske Mijnssen, direction musicale Christophe Rousset, décors Herbert Murauer, costumes Julia Katharina Berndt, lumière Bernd Purkrabek, Opéra du Rhin en coproduction avec l'Opéra National de Lorraine, saison 2018-2019.

35. Louis Jouvet, Préface à Pratique pour fabriquer scènes et machines de théâtre par Nicola Sabbattini, op. cit., p. XXXVII.

36. L'exemple donné fait état d'une création d'opéra mais cette étape de construction se retrouve également durant la création d'une représentation théâtrale.

37. Jean-Luc Mattéoli, « L'objet pauvre dans le théâtre contemporain », Images Re-vues [En ligne], $\mathrm{n}^{\circ}$ 4, 2007, URL de référence : http://journals.openedition.org/imagesrevues/125; consultée le 07 octobre 2020 .

38. Ibid.

39. Ibid.

40. Élie Konigson, «Les objets de représentation au théâtre (XVème - XVIIème siècle) ", Nouvelle Revue du XVIe Siècle, Vol. 14, n 2, Librairie Droz, Paris-Genève, 1996, p. 189-199.

41. Élie Konigson, « Espaces dramatisés », Ligeia, vol. 73-76, nº 1, 2007, p. 82.

42. L'objet conserve la disposition matérielle conventionnelle d'une missive. L'émetteur est désigné sur la droite comme : «Absolute Nonsense / 10 Fortyfive Am / On a Tuesday Morn / [...] ». Nous effaçons volontairement le nom de la ville du théâtre marquée par le [...], il ne faudrait pas que le pfüsch ne dénonce de trop son auteur...

43. "Dear pure invention, / it is with the utmost apathy that I find myself standing in the Running Props office of the Theatre [...] writting this most uninteresting of letters, which says nothing, means nothing and in years to come to will have no bearing on anything whatsoever. / I will remain your obedient servant until such times as my contract of employment comes to an sad- four days! / Absolute Nonsense. » [traduction personnelle]

44. L'accessoiriste n'étant pas tout à fait historien, il choisira aussi ce qui semble pour le public correspondre à une époque (sauf si la demande du metteur en scène ou du scénographe l'indique). L'illusion d'une écriture à l'ancienne semble parfois assez grotesque mais le vrai n'est pas toujours réaliste, comme le signale Léon, le machiniste de Louis Jouvet : « Léon un jour a dit, en contemplant une statue véritable qu'on avait apporté sur la scène : "Ça ne fait pas vrai" » dans Louis Jouvet, Préface à Pratique pour fabriquer scènes et machines de théâtre par Nicola Sabbattini, op. cit., p. XXXIV. 
45. Le lorem ipsum ou faux-texte est une convention de la fabrique éditoriale, et désigne génériquement une suite de mots latins (empruntés à Cicéron) utilisée de façon provisoire pour calibrer une mise en page.

46. " Now, I've written the whole thing : for Christ's sake give me a drink », " Thank God, it will soon be dark ", " the parchment is hairy », " oh my hand ", d'après Michael Camille, Images on the Edge: The Margins of Medieval Art, Reaktion Books, 1992, [traduction personnelle].

47. «Ce qu'exprime le bricolage, c'est un désir fort de renouer avec soi-même, le désir que ce soit le travail qui fasse partie de la vie et non pas la vie qui soit sacrifiée au métier » dit aussi PierreFrançois Dupont-Beurier, Petite philosophie du bricoleur, Paris, Milan, 2006, p. 104.

48. C'est aussi le reproche fait au kludge ou au quick-and-dirty. La rapidité de l'exécution entraîne paradoxalement une certaine lenteur si l'on doit réintervenir dessus.

49. Nous renvoyons le lecteur et la lectrice à la définition de la mètis dans l'ouvrage de Marcel Détienne \& Jean-Pierre Vernant, Les ruses de l'intelligence, la mètis des Grecs, Paris, Flammarion, 2009, p. 10 : «La mètis est bien une forme d'intelligence et de pensée, un mode du connaître ; elle implique un ensemble complexe, mais très cohérent, d'attitudes mentales, de comportements intellectuels qui combinent le flair, la sagacité, la prévision, la souplesse d'esprit, la feinte, la débrouillardise, l'attention vigilante, le sens de l'opportunité, des habiletés diverses, une expérience longuement acquise ; elle s'applique à des réalités fugaces, mouvantes, déconcertantes et ambiguës, qui ne se prêtent ni à la mesure précise, ni au calcul exacte, ni au raisonnement rigoureux. Or, dans le tableau de la pensée et du savoir qu'ont dressé ces professionnels de l'intelligence que sont les philosophes, toutes les qualités d'esprit dont est faite la mètis, ses tours de main, ses adresses, ses stratagèmes, sont le plus souvent rejetés dans l'ombre, effacés du domaine de la connaissance véritable et ramenés, suivant les cas, au niveau de la routine, de l'inspiration hasardeuse, de l'opinion inconstante, ou de la pure et simple charlatanerie ".

50. Michel De Certeau, L'Invention du quotidien, Arts de Faire, T.1, p. XLVI.

51. Ibidem.

52. Ibidem.

53. Cette notion fait écho à celle d' « espace » imaginé par le philosophe. Celui-ci distingue le lieu, stable, " en propre » de l'espace, du « lieu pratiqué », compris comme les opérations qui y sont affectés. À la différence d'une description et d'une analyse segmentante comme le fait le lieu, l'espace offre un récit traversant et expérientiel.

54. Michel De Certeau, L'Invention du quotidien, op. cit., p. XXXVI.

55. Le Tour d'écrou (The Turn of The Screw) de Benjamin Britten, mise en scène par Robert Carsen, a été créé à Berlin en 2011.

56. Jacques Copeau cité par Jean-Loup Rivière, "L'art de l'acteur ", dans Le Théâtre français du XXème siècle, Robert Abirached (dir.), L'Avant-scène théâtre, 2011, p. 462- 463.

57. On retrouve l'image du gaffer du technicien. Thomas Hirschhorn, Stand-alone, exposition personnelle au Arndt \& Partner, Berlin, 2007 et à The Mistake Room, Los Angeles, 2016. Jordi Colomer, « X VILLE », Production : LOOP Barcelona, Xarxa de Centres d'Arts Visuals de Catalunya, Arts Santa Mònica (Barcelona), Jardins-Fabriques d'Annecy, Fondation Sandretto Re Rebaudengo de Turin, and CO Productions Barcelone-Paris, 2015.

58. Nicolas Bourriaud, «Qu'est-ce que le réalisme opératif?", Catalogue de l'exposition Il faut construire l'hacienda, textes de Éric Troncy et Nicolas Bourriaud, Tours, CCC, 1992.

59. On citera par exemple: Tremblez Machines! et Animal épique, de Jean-Pierre Larroche, Catherine Pavet et Zoé Chantre, 2018.

60. Entre autres : Forbidden di Sporgersi de Pierre Meunier et Marguerite Bordat, 2015.

61. Thomas Golsenne, «Les chaînes opératoires artistiques ", art. cit.

62. André Leroi-Gourhan, Le Geste et la Parole, Vol. II, La Mémoire et les rythmes, Paris, Albin Michel, 1965. 


\section{RÉSUMÉS}

Le pfüsch est un terme alsacien qui désigne le travail bâclé. Utilisé dans les coulisses de l'Opéra du Rhin, il nomme ces bidouilles grossières faites dans l'urgence du spectacle. À partir de ce mot régionaliste, pourrait-on voir se dessiner, davantage qu'un raté, une technique qui fait se rejoindre le bricolage défini par Claude Lévi-Strauss et le braconnage caractérisé par Michel De Certeau ? Pourrait-on voir dans le pfüsch non pas seulement une déviation du modèle exemplaire, sujet à reproche, mais un outil de construction de la scène qui rend compte de deux de ses fondements, la visibilité et la précarité ?

INDEX

Mots-clés : scénographie, bricolage, tactique, ruse, technique, visibilité, précarité

\section{AUTEUR}

\section{CLÉMENTINE CLUZEAUD}

Scénographe et doctorante, Clémentine Cluzeaud prépare une thèse intitulée « La scénographie peut-elle faire œuvre pour elle-même ? » sous la direction de Sandrine Dubouilh (laboratoire CLARE de l'école doctorale Montaigne Humanités - Bordeaux). Elle travaille régulièrement à l'opéra du Rhin en tant qu'accessoiriste plateau. 